Revista Educación

ISSN: 0379-7082

ISSN: 2215-2644

revedu@gmail.com

Universidad de Costa Rica

Costa Rica

\title{
Límites y posibilidades de la mejora escolar en el contexto patagónico chileno: el caso de las escuelas de la Comuna de Coyhaique, Región de Aysén, Chile ${ }^{[1]}$
}

Retamal Cisterna, Silvia Natalia; Gairín Sallán, Joaquín

Límites y posibilidades de la mejora escolar en el contexto patagónico chileno: el caso de las escuelas de la Comuna de Coyhaique, Región de Aysén, Chile ${ }^{[1]}$

Revista Educación, vol. 44, núm. 2, 2020

Universidad de Costa Rica, Costa Rica

Disponible en: http://www.redalyc.org/articulo.oa?id=44062184031

DOI: https://doi.org/10.15517/revedu.v44i2.39821

Esta obra está bajo una Licencia Creative Commons Atribución-NoComercial-SinDerivar 3.0 Internacional. 


\title{
Límites y posibilidades de la mejora escolar en el contexto patagónico chileno: el caso
} de las escuelas de la Comuna de Coyhaique, Región de Aysén, Chile ${ }^{[1]}$

\author{
Limitations and Possibilities for School Enhancement in a Chilean Patagonia Context: Coyhaique Community \\ schools in the Aysén Region, Chile
}

Silvia Natalia Retamal Cisterna

Universidad de Los Lagos, Chile

silvia.retamal@ulagos.cl

(D) http://orcid.org/0000-0001-6515-8501

Joaquin Gairin Sallán

Universidad Autónoma de Barcelona, España

joaquin.gairin@uab.cat

(iD http://orcid.org/0000-0002-2552-0921

\author{
DOI: https://doi.org/10.15517/revedu.v44i2.39821 \\ Redalyc: http://www.redalyc.org/articulo.oa?id=44062184031
}

Recepción: 22 Enero 2020

Aprobación: 09 Junio 2020

\section{RESUMEN:}

El presente artículo ilustra los límites y las posibilidades de los procesos de cambio en las escuelas municipales de la comuna de Coyhaique, en la Patagonia chilena. Utilizando los constructos teóricos sobre la mejora escolar y las experiencias de docentes/ directivos de la comuna de Coyhaique, se efectúa un análisis cualitativo sobre los procesos de cambio que se desarrollan en dichas instituciones educativas al momento de implementar una mejora educativa, en este caso concreto, la intervención de un programa de formación docente y competencias directivas[2] (Proyecto AECID Coyhaique). El objetivo de este trabajo es mostrar los condicionantes institucionales y contextuales que la escuela, a través de directivos y docentes, valora como importantes para lograr un cambio educativo. Los datos provenientes de entrevistas a directivos/docentes de nueve escuelas municipales de Coyhaique, ejecutadas desde un enfoque socioantropológico describen las características locales cuando se habla de mejora institucional y la distancia existente respecto de las teorías que explican el cambio educativo. El análisis concluye que existen factores los cuales favorecen los procesos de cambio en las organizaciones educativas, que son comunes también para las escuelas situadas en el contexto patagónico, tales como: relevar los procesos y resultados de la enseñanza como foco principal, propiciar espacios para la reflexión compartida, pensar la institución educativa como un lugar de organización y formación del profesorado, y abrir la escuela hacia el exterior. Sin embargo, sus efectos varían en la medida que interactúan con otras variables contextuales como, la situación geográfica, las condiciones socioculturales y la rigidez de la normativa chilena en materia educativa.

Palabras ClaVe: Cambio educativo, Mejora escolar, Cambio organizacional, Programas de desarrollo educativo, Educación rural.

\section{ABstRACT:}

This article shows the limitations and possibilities involved in enhancing schools in the municipality of the Coyhaique commune in Chile's Patagonia Region according to theoretical constructs on school enhancement and the experience of teachers/ principles working in the Coyaique commune. A qualitative study was conducted on transformation processes at schools in the aforementioned area for educational improvement purposes, specifically consisting of a teacher training and school principal skills intervention program[3] (AECID Coyhaique Project). The objective of this study is to look at the institutional and contextual conditioning factors at schools that principals and teachers consider to be important for educational transformation. Data is based on interviews with principals/teachers of nine municipal schools in Coyhaique which were conducted using a socioanthropological focus. The data reveals a chasm between local understanding of school improvements and theoretical explanations regarding educational transformation. The study concludes that there are factors favoring transformation processes at schools that are also common to schools in Patagonia, such as: redirect the focus on teaching processes and results, provide opportunities for shared discussion, rethink school as a place to organize and train teachers and make schools more accessible to outsiders. However, results vary, to that extent, that they interact with other contextual variables, such as geographic location, sociocultural conditions and inflexibility of Chilean educational policies. 
KEYWORDS: Educational Transformation, School Enhancement, Organizational Change, Educational Development Programs, Rural Education.

\section{INTRODUCCIÓN}

En este trabajo se presentan perspectivas teóricas y aplicadas las cuales explican el cambio educativo para la mejora escolar en las escuelas municipalizadas de la comuna de Coyhaique. Esto se efectúa por medio del análisis de experiencias docentes/directivas[4] que determinan los límites y las posibilidades del cambio educativo en dicho territorio. Las aportaciones aquí descritas revelan la existencia de ciertas nociones contextuales que no están contempladas en las conceptualizaciones teóricas habituales sobre la mejora educativa y que forman parte del contexto local y la cultura escolar de la comuna de Coyhaique. Este trabajo consta de cuatro partes, la primera consiste en presentar brevemente los aspectos más significativos de la teoría del cambio educativo, la segunda, se centra en acotar la parte metodológica, cuyos datos forman parte de un trabajo de investigación doctoral desarrollado en las escuelas municipales de Coyhaique. La tercera parte, corresponde a los resultados que describen la relación entre los condicionantes planteados por las teorías del cambio educativo y la realidad descrita por docentes/directivos (es importante aclarar que, en el caso de las escuelas rurales observadas, existe la modalidad unidocente, razón por la cual, el profesorado ejecuta la función directiva), estableciendo, los límites y las posibilidades que tiene el cambio educativo en dicho territorio. Y por último, las reflexiones y conclusiones del trabajo presentado.

El fundamento principal de la teoría del cambio educativo se basa en la asunción de determinados principios y valores tales como la colaboración, la participación, el compromiso institucional y un consenso sobre la coordinación de tareas, necesarios para generar cambios en los centros educativos (Miranda, 2002). La bibliografía acumulada respecto al cambio educativo (Fullan, 2002a, 2002b; Escudero, 2001; Hargreaves, 2003; Santos, 2002, 2008; Gairín y Rodríguez, 2011) identifica algunos factores que lo favorecen, señalando elementos organizativos claves para lograr la mejora, pero también denotan un alto consenso sobre los mecanismos contextuales, que afectan fuertemente los procesos de enseñanza y aprendizaje los cuales apuntan principalmente a las condiciones socioculturales del alumnado del sector público, en muchos casos desfavorable, como la segregación y la inequidad, que en el caso chileno se encuentran profundamente arraigados (Atria, 2009).

Los procesos de cambio educativo en las escuelas, convienen ser estudiados y abordados desde su entorno más próximo (Mertler, 2016 y Mora, Monge y Santiago, 2018). El contexto donde se desarrolla la vida escolar es el escenario donde la práctica del cambio mejor se representa. Como bien señala Murillo (2011) en un estudio orientado hacia la eficacia escolar:

Solo analizando los procesos que ocurren en las escuelas e integrando este conocimiento con el saber acumulado por la investigación será posible establecer criterios, pautas y procedimientos adecuados de avaluación que nos lleven a la eficacia y calidad de los centros educativos y de los programas que se desarrollan en ellos. (Murillo, 2011, p.53)

La región de Aysén, donde se sitúan las escuelas de la comuna de Coyhaique, presenta algunas características educativas que le son propias y que la posiciona como una de las más vulnerables del país, con altas tasas de reprobación y abandono (Ministerio de Educación de Chile [MINEDUC], 2008), bajos niveles de alfabetización (Ministerio de Desarrollo Social de Chile, 2011) y una considerable disminución de matrículas en el sector público (PADEM, 2011). Pese a esto, la función social de la escuela debiera centrar sus esfuerzos en contrarrestar estas carencias de origen traducidas en segregación e inequidad social, a través del análisis y la detección de las prácticas nocivas que se efectúan dentro de estas (Murillo, 2011).

En materia de desarrollo investigativo, hay poca evidencia sobre investigaciones cualitativas que relacionen el desarrollo educativo con el contexto social patagónico. La herramienta más utilizada, pero insuficiente, que existe como referente para medir los logros educativos en ese contexto corresponde a la prueba SIMCE 
(Sistema de medición de la calidad de la enseñanza) y bajo este parámetro se desarrollan la mayoría de los acuerdos educativos en el contexto nacional chileno (Raczynski y Salinas, 2008). Las políticas educativas nacionales se basan más en la evidencia internacional que en la investigación educacional propia y de la realidad de las escuelas de Chile (OCDE, 2004), opción que puede explicar los principales fracasos en los resultados de los programas diseñados para la mejora por no considerar ni los contextos, ni la complejidad en las que se enmarca el cambio educativo (Tiana, s.f.).

La presente aportación muestra algunas de las dinámicas escolares propias de las escuelas de la comuna de Coyhaique que pone en evidencia las rupturas entre los marcos teórico-prácticos que analizan la institución educativa y la interacción de la escuela con un entorno social complejo.

\section{Aspectos relevantes Para la Mejora EDUCATIVA}

A lo largo de la historia, la institución escolar ha estado fuertemente cuestionada. Tras el surgimiento de los movimientos que van en la línea de la desescolarización, se han instalado diversas críticas respecto a la función y al aporte que realiza la escuela a la sociedad. Una de las críticas que ha tenido más presencia en el ámbito investigativo apuntan a las teorías de la reproducción cultural cuya base argumentativa está en situar al sistema escolar como un mero reproductor de las relaciones de poder existentes en la sociedad y un distribuidor del capital cultural entre las clases sociales (Bourdieu y Passeron, 1996) al considerar que los códigos escolares responden a un estilo de enseñanza que solamente descifran y comprenden los integrantes de ciertas clases sociales, principalmente las más cercanas a los poderes políticos y económicos, que hace que los niños más pobres requieran un doble esfuerzo dentro de la escuela para alcanzar logros educativos. A partir de estas críticas, surgen también otras perspectivas teóricas, que consideran la escuela como un espacio de transformación social, ampliando la mirada investigativa al cuestionarse las condiciones que pueden hacer que la escuela llegue a ser más eficaz y equitativa (Bürgi y Peralta, 2011). Sobre la base de este cuestionamiento, comienzan a plantearse una serie de ideas que engloban el cambio educativo.

El objetivo de las teorías del cambio es lograr que los propios establecimientos educacionales creen conocimiento y formen comunidades de aprendizaje. Más allá de las teorías que se centran "en comprender cómo las relaciones de poder externas son transmitidas por el sistema" (Bernstein, 1990, p. 20), es necesario describir cómo la escuela en su conjunto aborda ciertos procesos en la toma de conciencia sobre aquellas prácticas que conducen a transformar la realidad educativa. El centro escolar se convierte, en este contexto, en una instancia de aprendizaje.

Para Gairín (2015), la mejora en las instituciones es “parte consustancial al proceso organizativo” (p. 17) y, para conseguirla, sugiere un análisis sobre los factores que facilitan u obstaculizan el cambio pretendido. Sitúa el foco en los procesos de autorrevisión, planificación y acción estratégica, primando la cultura colaborativa a través del intercambio de experiencias y el aprendizaje profesional dialógico. Esta concepción no solo reclama a las y los profesores y escuelas la asunción de nuevas tareas y cometidos, sino también la asunción de unos determinados principios y valores para desarrollarlas: colaboración, participación, compromiso institucional, negociación y consenso (Miranda, 2002). Por otra parte, Escudero (2001) concibe la mejora como un aprendizaje y como tal requiere de ciertos procesos y estrategias de trabajo altamente definidos y organizados para propiciar la eficacia de esa interacción. Fullan (2002a) plantea que "los profesores y directores deben moverse hacia el peligro y correr el riesgo de unirse al medio formado por los padres y la comunidad, la tecnología y el gobierno, si quieren tener éxito" (p. 10). Es decir, asumir el cambio en forma de acciones concretas.

La estructura autocrática que tiene el sistema educativo chileno y su relación con la comunidad educativa de un territorio, provoca que los principales implicados en los procesos de cambio sean considerados como entes pasivos, como meros receptores del cambio, sin la implicación colectiva de sus miembros y sin una participación concreta. Las innovaciones se urden mayoritariamente fuera de las paredes de la escuela, 
olvidado los aspectos participativos y cooperativos. Así se produce un desgaste de los profesionales que la conforman al mismo tiempo que las escuelas restan esfuerzos en crear e idear estrategias para abordar estos complejos procesos. En este contexto, se describen a continuación los cuatro condicionantes claves considerados en el estudio y que representan ideas que a menudo se repiten, debaten y se promueven en la literatura sobre el cambio educativo, estos son: procesos y resultados de la enseñanza-aprendizaje como foco principal; espacios para la reflexión compartida; la escuela como instancia para la formación docente y la escuela abierta al exterior.

\subsection{Procesos y resultados de la enseñanza-aprendizaje como foco principal}

La teoría sobre la mejora escolar plantea que todo proceso de mejora escolar debe conducir a provocar cambios en los progresos y resultados de la enseñanza (Guarro, 2005; Bolívar, 2004 y Escudero 2001). Las estrategias, de cualquier índole que se apliquen en la institución, deben estar encaminadas a producir mejoras en los aprendizajes del estudiantado. Las escuelas tienen la difícil misión de dar solución a diversas demandas socioculturales, pero, aun así, no deben perder de vista su principal función: avances en materia de resultados del aprendizaje. Cuando se habla de resultados, no solo se hace referencia a los resultados de la aplicación de pruebas estandarizadas o evaluaciones calificadas, sino también a los avances cualitativos que ha tenido el alumnado. Es necesario cuestionar y debatir qué aprendizajes deben ser perseguidos y sobre qué y cómo se deben evaluar los dispositivos que pone en juego la enseñanza. Los resultados deben analizarse con miras a la comprensión de todo lo que se ejecuta en la escuela. Como expone Escudero (2001):

Desde los resultados escolares, entendidos en su acepción amplia e intensa, habrá que activar la crítica y la reflexión a la

luz de los ideales pedagógicos, y su traducción en cuestiones concretas y específicas de forma tal que sean aptas para activas decisiones y prácticas sucesivas. (p.40)

Según Guarro (2005), el verdadero significado del cambio, en coherencia con su propósito moral, debe ser la mejora de los aprendizajes de todo el alumnado. Si no mejoran los resultados no se puede decir con propiedad que se haya producido un cambio.

\subsection{Espacios para la reflexión compartida}

Una idea que expone Hargreaves (2003) es la siguiente:

Rara vez pasa una semana sin que las escuelas se vean frente a nuevas imposiciones de cambios interminables. Mi investigación anterior, como las de muchos otros, mostró que trabajar más estrechamente con los colegas puede servir para evitar la duplicación de los esfuerzos, compartir la carga de trabajo, brindar apoyo moral y dar a los docentes la fuerza colectiva necesaria para establecer prioridades entre todas las demandas que se les plantean. (Hargreaves, 2003, p.30)

Una forma de hacer escuelas para el aprendizaje colectivo debiera sustentarse en el diálogo, la reflexión y la toma consciente de decisiones. La institución escolar es un lugar donde convergen, además de la acción educativa, una multiplicidad de miradas, ideologías y diversas formas de pensar. Por ello, uno de los ejes principales que sostiene la mejora educativa radica en la capacidad de generar espacios de reflexión colectiva sobre las prácticas educativas. La participación vinculante en estos espacios está asociada no solo a la voluntad de querer intervenir o aportar para la mejora, sino que también va unida a la capacidad de las instituciones escolares de contar con espacios físicos y temporales para dichas prácticas. A este respecto ya se refería Santos (2002):

Obsérvese que hay tiempos profesionales destinados a la planificación, muchos dedicados a la acción y muy pocos que se destinen a la reflexión sobre la acción. La estructura del trabajo en los centros, basada en la fragmentación de los saberes, en la fragmentación de espacios y tiempos, lleva a una visión individualista de los aprendizajes. (p.104) 
Este mismo autor señala que una concepción individualista de la realidad lleva a planteamientos de naturaleza escasamente cooperativa. En este punto, el concepto de comunidad educativa cobra relevancia en el sentido que se comprenda como el resultado del trabajo colaborativo en la escuela. "Se trata de superar etapas y de pasar de conglomerados humanos al fortalecimiento de equipos educativos y a la creación de comunidades profesionales y comunidades educativas que funcionan como comunidades de aprendizaje" (Gairín y Rodríguez, 2011, p. 40). A este respecto, los espacios para la reflexión colectiva serán plausibles en la medida que utilicen herramientas adecuadas que permitan el trabajo efectivo y no solo porque las personas compartan espacios, tiempo e interés.

\subsection{La escuela, una instancia de formación docente}

Santos (2002) destaca una cuestión fundamental para propiciar la mejora escolar: la formación en el centro. Esta modalidad de formación considera a la escuela como una unidad funcional de planificación, acción, evaluación y cambio, y como un excelente medio para que los profesionales alcancen una comprensión enriquecedora que lleve a la transformación de la práctica. Si la formación queda reducida a iniciativas personales, si cada profesor acude a los cursos o efectúa las actividades de manera individual, el enriquecimiento colectivo no existe:

Para ello hace falta que la iniciativa surja del centro, que la compartan todos o la mayoría de los profesionales, que se cuente con expertos externos, que esté encaminada a la mejora de la práctica profesional a través del aprendizaje que realizan los profesores. (Santos, 2002, pp.105-106)

Considerar la institución escolar como un lugar de formación para sus profesionales se relaciona con elevar la producción de conocimiento del propio establecimiento; también, con que el profesorado logre un mayor grado de profesionalización abandonando el mero rol técnico reproductor de decisiones curriculares determinadas en otros contextos. Como señala Sánchez (2009), refiriéndose a la necesidad de las escuelas de transformarse en lugares de aprendizaje para sus docentes, deben "abandonar el estricto sentido burocrático que en la gran mayoría de ellas impera, para transformarse en espacios de abierta colaboración, lo que permitirá la existencia de debates, análisis, diálogos y discusiones de las prácticas docentes” (p. 61). Abrir espacios para la formación en las escuelas, no necesariamente compromete solo al cuerpo docente, más bien, a toda la comunidad educativa, en la facilitación de los entornos y los tiempos otorgados por la misma institución, pues solo así se justifica la necesidad de abrir espacios y crear canales de comunicación más eficientes entre los diferentes estamentos.

\subsection{Escuela abierta al exterior}

Según la literatura sobre la mejora escolar, una escuela que interactúa con otras escuelas y con el mundo exterior es una organización que goza de buena salud institucional. Ciertamente, los procesos de cambio deben llevarse a cabo en cada escuela y en cada aula, pero no llegaran a impactar en la comunidad escolar si no tienen un sentido social del cambio. Guarro (2005) señala que es imposible sobrevivir cambiando aisladamente porque las escuelas necesitan sentirse partícipes de un proyecto más amplio y formar parte de otras instituciones escolares, que propicien aprendizajes e intercambien experiencias.

Si docentes entre sí y directivos se muestran dispuestos a colaborar con otras personas, se generan renovadas dinámicas en la institución escolar, más abiertas, más inclusivas y capaces de generar confianza hacia el resto de comunidades educativas (Escudero, 2001). Esto motiva también a las familias a participar, a opinar, a colaborar y sentirse atraídos por los diversos proyectos escolares. Haciéndose eco de las palabras de Lieberman y Miller, 1984 en Krichesky y Murillo (2011): 
Con tanta gente comprometida en una misión compartida por tantos, en un espacio y un tiempo tan compacto, es tal vez una de las mayores ironías -y una de las grandes tragedias de la enseñanza- que tanto trabajo se desarrolle en un aislamiento profesionalmente consagrado. (p.66)

Considerar la escuela como una unidad básica de acción, cohesionada internamente, es beneficioso para quienes la conforman, pero si no abre una puerta al exterior está condenada al aislamiento.

\section{CONSIDERACIONES METODOLÓGICAS}

Para este trabajo se utilizaron los datos obtenidos de una investigación doctoral desarrollada en la comuna de Coyhaique, en la Patagonia Chilena, cuya investigación se planteó desde un enfoque socioantropológico, tomando herramientas de la etnografía para analizar el ámbito socioescolar correspondiente al conjunto total de escuelas municipalizadas de la comuna de Coyhaique. Se analizaron nueve instituciones a través de técnicas etnográficas: observación participante, entrevistas y análisis de documentos, con énfasis en los testimonios obtenidos en las entrevistas organizadas al grupo directivo, dada la responsabilidad y representación que asumen frente al cambio y al desarrollo organizacional de los establecimientos (Gairín y Muñoz, 2008). Las técnicas que se utilizan para la etnografía educativa proporcionan una descripción más profunda sobre el funcionamiento de las instituciones escolares, es decir, sobre lo que sucede dentro de las escuelas (Serra, 2004). Esta perspectiva investigativa resulta útil a la hora de comprender el contexto y la cultura donde se genera la mejora educativa. El trabajo con las escuelas se desarrolló en un periodo de inmersión de cuatro meses, no consecutivos. El análisis de la información se hizo en base a las teorías del cambio educativo señaladas en el punto anterior, las cuales permitieron describir e interpretar: las posibilidades o límites que tienen ciertos aspectos declarados en la literatura sobre la mejora escolar y, a través de los relatos, el significado que tiene para las personas los procesos de cambio educativo.

\section{Principales resultados}

Los resultados describen e ilustran la relación entre los condicionantes del cambio descritos en la teoría, la realidad de las escuelas de Coyhaique y el significado que adquieren para quienes conforman la escuela en este contexto, permitiendo así comprender las dinámicas escolares cotidianas que determinan el cambio educativo en un territorio determinado.

\subsection{Procesos y resultados de la enseñanza y el aprendizaje como foco principal}

La mayoría de las innovaciones y proyectos de mejora de los establecimientos educativos de la comuna de Coyhaique apuntan al fortalecimiento de los procesos de enseñanza y aprendizaje, enfocados a incrementar los resultados académicos en términos cuantitativos. La preocupación constante por la excelencia académica vuelca la toma de decisiones y las adaptaciones hacia los criterios exigidos en las pruebas estandarizadas que miden la calidad de la enseñanza. Por tanto, el trabajo técnico pedagógico se refleja en el refuerzo de los planes de lectura y matemática, que incurre en una serie de mecanismos para adaptar la planificación curricular y la capacitación específica solo a algunos contenidos de alta valoración en el Sistema de Evaluación de la Calidad de la Enseñanza (SIMCE).

A pesar de este requerimiento, que constriñe la libertad en la planificación y repercute en los modos de enseñanza, la apuesta de las escuelas de la comuna de Coyhaique se enfoca en mantener un cierto rendimiento académico que estratégicamente habilite a estudiantes en la participación de otros espacios de desarrollo personal. Impulsan así proyectos de innovación, como, por ejemplo, la orquesta juvenil, que permite el aprendizaje y la práctica de un instrumento musical dentro del periodo escolar, al mismo tiempo 
que anima a obtener un cierto rendimiento académico para poder participar en dicha actividad, así motivar al estudiantado a lograr un buen rendimiento académico. En el caso chileno, cuando las metas escolares, medidas como resultados de aprendizaje, no son alcanzadas, la ley estima drásticas medidas que pueden incluso decretar el cierre del establecimiento (Vera, Salvo y Zunino, 2013).

Se cuestiona constantemente el enfoque castigador de las evaluaciones, que ha desembocado en una pérdida de interés del personal docente por contextualizar los aprendizajes, argumentando que con esta evaluación no ganan nada porque ya tenían ganado el título de los peores resultados regionales. A pesar de las controversias relacionadas con las pruebas SIMCE, las escuelas no quieren perder de vista el enfoque constructivo que debe tener la evaluación de los aprendizajes en el aula. Así, la reducción de pruebas objetivas es reemplazada por otras modalidades de evaluación, como los portafolios en el aula, que ofrecen una forma más colaborativa de evaluar los aprendizajes, aunque su implementación esté condicionada a procesos y recursos que van ligadas también a otras estrategias pedagógicas. En palabras de algunas de las personas entrevistadas: "lo ideal es trabajar la evaluación con el portafolio, y para mí esa sería una escuela ideal” (Entrevista 4, comunicación personal, 18 de junio de 2009); “cada taller que ellos (docentes) practiquen con sus alumnos/as y aporten la evidencia de que lo practicaron, va a ser una forma de evaluarlos" (Entrevista 3, comunicación personal, 17 de junio de 2009).

Por otra parte, la poca conexión que existe entre el currículum oficial y la realidad de la comuna es otro de los desafíos al que deben enfrentarse las escuelas de la comuna de Coyhaique. Los planes y programas de estudios no están contextualizados, pese a que existe la posibilidad de adaptación curricular a través de un proyecto educativo institucional, señalando "que el trámite de aprobación es un tanto engorroso para la escuela, labor que ellos no están dispuestos a asumir" (Retamal, 2012, p.177). También existen casos como el de una escuela que trabaja con planes y programas de estudio propios y que finalmente fue aprobado por el MINEDUC. El relato de una directora ilustra el proceso vivido de la adaptación curricular:

Nosotros tuvimos necesariamente que hacer una fuerte autocrítica no solo al sistema y a la normativa, sino también a lo que estábamos haciendo. Esto marcó un antes y un después en la escuela porque fue súper duro y hubo quienes nos molestamos en un momento. Pasó de todo. (Entrevista 8, comunicación personal, 15 de junio 2010)

También son conscientes de que los resultados de la enseñanza marcarán una diferencia en el futuro; por eso, ponen mucho énfasis en la extensión de estudios de sus estudiantes para incrementar sus posibilidades laborales. Un director comenta sobre sus estudiantes:

Yo les digo, ¡claro! ustedes pueden cortar leña, pero corten su leña. Si ustedes estudian, sacan octavo y después sacan su licencia de conducir, ya no tienen que cortarles la leña a otros, ustedes pueden ir a vender su propia leña a Coyhaique. (Entrevista 7, comunicación personal, 14 de junio 2010)

\subsubsection{Después de la escuela ¿qué?}

Dadas las características demográficas y geográficas del entorno donde se sitúan las escuelas de Coyhaique, la vinculación y el compromiso de estas con el territorio es más significativa. Docentes y directivos desarrollan una labor que va más allá de la etapa escolar, situando las expectativas en la continuación de estudios, en la inserción al campo laboral y en preparar buenos ciudadanos/as para la comuna. La motivación se centra fuertemente en mantener y fortalecer el sistema educativo público, que se encuentra altamente estigmatizado desde hace algunas décadas.

Una característica que adopta la gestión escolar en estos territorios es el fortalecimiento de aquellos ámbitos donde la escuela contribuye al desarrollo regional. Tarea nada fácil, ni reducida solo al espacio de la enseñanza. La escuela en estos territorios es vista como un fuerte motor de desarrollo regional, cuyo nexo se $\mathrm{da}$, concretamente, estableciendo un puente entre estudiantes de enseñanza secundaria y el campo laboral. 
$\mathrm{Al}$ respecto, un director señala: "todas esas especialidades las hemos enfocado hacia el desarrollo social y económico de la comuna y así tenemos dos especialidades, que son mecánica automotriz y también la especialidad de ventas" (Entrevista 9, comunicación personal, 16 de junio 2010). Las expectativas en el desarrollo de la región deben concretarse en brindarle al alumnado oportunidades y confianza en sus capacidades para continuar en el mundo laboral local, como pone de manifiesto una directora:

Nosotros como establecimiento estamos entregando ciudadanos que aportan a la comunidad su preparación, su comportamiento, y que, además, son capaces de desarrollar un proyecto de vida, elaborarlo y ejecutarlo en todos sus ámbitos: personal, familiar, etc. Por ejemplo, los chiquillos egresados que tienen sus trabajos, un buen trabajo, permanecen en él, hacen familia propia, optan y postulan a su casa y van desarrollándose como cualquier ciudadano. Nosotros creemos que ese es un buen aporte a la comunidad: no tener chiquillos que van a estar permanente subsidiados, permanentemente siendo un lastre para el Estado, dependiente de todos los problemas sociales. (Entrevista 8, comunicación personal, 15 de junio 2010)

En este sentido, la reflexión debiera apuntar a discutir el rol que tienen las escuelas en ciertos territorios, predominantemente orientado a frenar la deserción, la disminución de la pobreza, conectar con el campo laboral, formar personas de familia y acceder a beneficios estatales, entre otros. Podría considerarse un rol limitado, más bien conservador, que podría ser el de cualquier grupo de escuelas en otro lugar del país. Entonces, cabe cuestionar, cuál es el sello identitario de la comuna en cuanto a la formación escolar. En la medida en que las escuelas situadas en un territorio no cuestionan su significado, sus características particulares, las políticas locales, los conflictos con el espacio más global o nacional, que en muchos términos puede explicar la enorme brecha que existe entre las escuelas rurales y las urbanas, la educación pierde su sentido en estos espacios y a largo plazo puede transformarse en un instrumentos de desigualdades educativas, reflejadas en desarraigo y desadaptación producto de la migración desde un entorno rural a las escuelas de la ciudad. Las palabras de un director describen esta realidad:

Yo no tengo mucha claridad con respecto a qué va a pasar con las escuelas municipales, por lo que yo veo, hoy en día vamos a ser un ente en extinción, nos vamos a extinguir; es decir, con estos lineamientos nosotros vamos a desaparecer como escuelas municipales, nos vamos a transformar en escuelas particulares y lo digo sin desmerecer a las escuelas particulares, pero ellos tienen otro giro (Entrevista 7, comunicación personal, 14 de junio 2010).

La mayoría de las escuelas participantes tienen expectativas en sus estudiantes "que lleguen a lograr la mayor cantidad de competencias, habilidades, destrezas, en resumen, lograr capacidades” (Entrevista 9, comunicación personal, 16 de junio 2010). En otras palabras, antes que proyectar en ellos el futuro de una sociedad más justa, más equitativa y con más oportunidades, parten primero procurando que cumplan con la enseñanza obligatoria, evitando la deserción, lo que hará factible su incorporación al campo laboral.

A pesar de los esfuerzos de las escuelas por retener al estudiantado, existen altas tasas de deserción y abandono de estudios:

Ellos desertan, hay una tasa de deserción importante en el sector y yo tengo un montón de niños que andan ahí. Yo los veo todo el año fuera de la escuela, vagando, dando vueltas, pateando piedras y es porque han fracasado en sus estudios. Lo que nosotros queremos es que, hoy día, los chicos vengan y no se vayan hasta diciembre. (Entrevista 7, comunicación personal, 14 de junio 2010)

Un director habla de buenos productos para referirse a las/los buenas/os estudiantes y estos productos son los que mejorarán la cultura de la ciudad. Al respecto señala:

Si no hay una cultura en una ciudad, ¿quién va a querer venir a Coyhaique? Está lleno de puros flaites como se dice aquí, puros pandilleros. Hoy viene un turista y pasa por la calle y le roban la máquina fotográfica. El turista dice: ¡ah qué voy a ir a hacer a Coyhaique! (Entrevista 6, comunicación personal, 24 de junio de 2009)

Cada institución educativa promueve de diferentes formas el ingreso al campo laboral. Dentro de las escuelas municipales, hay una institución de educación especial que propicia la pronta inserción de sus estudiantes al mundo del trabajo, con la perspectiva de continuar formándose, ya sea por iniciativa propia o 
por la empresa que los contrate. Así mismo, algunos liceos han adaptado su enseñanza y poseen especialidades acordes con el campo laboral que ofrece la región y así aseguran el futuro ocupacional de los que van egresando. Podría decirse que, en estas escuelas, es más patente el nexo entre la formación y el campo laboral; también, los liceos brindan oportunidades para el alumnado que regresa, después de cursar algunos estudios previos. Dos testimonios que aluden a la posibilidad de continuar estudios superiores:

Las posibilidades de seguir estudios son poquísimas en este territorio[5]. Hay institutos técnicos solamente y, cuando el alumno aspira a ser un profesional de otro nivel, tiene que salir forzosamente de la región porque no ofrece las posibilidades de una educación superior de nivel profesional alto. (Entrevista 3, comunicación personal, 17 de junio de 2009)

Son muy pocos los que llegan a una carrera académica. La parte monetaria no alcanza y conseguir beca es complicado porque tiene que ser de 6 para arriba para poder postular a una beca y no la consigue el que tiene 5 o 4,9 y, entonces, pierde la oportunidad de beca. (Entrevista 5, comunicación personal, 22 de junio de 2009)

En efecto, se ve con menos optimismo la dificultad de continuar una carrera universitaria, por la situación económica del alumnado y las escasas oportunidades, considerando el alto costo en el desplazamiento y la manutención en otra ciudad del país.

\subsection{Espacios para la reflexión compartida}

A pesar de que existen instancias para organizar la enseñanza, a través de un proceso de planificación (Jornadas de Planificación) y otro para analizar los resultados del aprendizaje (Jornadas de Análisis de Resultados), estas son escasas y están comandadas desde la institucionalidad educativa central con el fin de supervisar las acciones y resultados alusivos al SIMCE. El conjunto de necesidades que presentan las escuelas de la comuna de Coyhaique no alcanzan a ser analizadas y atendidas en las modalidades rígidas y acotadas de reflexión ofrecidas; se requiere más tiempo y espacios también en el ámbito informal. Al respecto, se plantea la necesidad de contar con más tiempo para la interacción entre pares (la mayoría de las discusiones se hacen a la hora del café, los recreos, entre pasillos o al término de la jornada) y el apremio ha habilitado áreas de descanso dentro de la jornada laboral como espacios para reflexionar en torno a alguna situación.

\subsubsection{Estigmatización y precariedad del sistema municipal}

El sistema educativo chileno proporciona un financiamiento adicional a aquellas escuelas que tengan estudiantes de carácter prioritario, procedentes de familias con más bajos ingresos económicos de la comuna, entre otros indicadores sociales. Este financiamiento se otorga a los establecimientos comprometidos con el desarrollo de los planes de mejoramiento institucionales para la eficacia de los aprendizajes del alumnado más vulnerables.

De las nueve escuelas consultadas, ocho de ellas han sido favorecidas con este beneficio variando los índices de vulnerabilidad. Las que concentran un número importante de estudiantes vulnerables (70\%) son precisamente las que señalan que el trabajo en este contexto es tremendamente complejo, pues no solo tiene que compensar las carencias económicas de las familias, sino que también tiene que atender problemas de orden psicológico y afectivo. Como señala el relato de un directivo cuya escuela atiende a un gran porcentaje de alumnos/as vulnerables:

Nosotros tenemos hijos de obreros y no hay hijos de profesionales. Son hijos de hogares monoparentales, o está la mamá, o está el papá, o está la abuelita, o está el tío, ya que las mamás por lo general trabajan como asesoras del hogar en Puerto Chacabuco y los niños quedan solos en la noche. Entonces, la parte afectiva la aborda la escuela, porque en el fondo este tipo de escuela tiene que hacerse cargo de la responsabilidad familiar, los problemas de hábitos, de buenas costumbres, de puntualidad, de la higiene personal, de todo se hace cargo el profesor jefe, principalmente el profesor jefe y todos los que trabajan al interior del establecimiento. (Entrevista 9, comunicación personal, 16 de junio 2010) 
Otro problema que aqueja a las y los directivos son los resultados del SIMCE y la no consideración de los índices de vulnerabilidad de las escuelas, lo que provoca que estas obtengan los más bajos puntajes pese a los esfuerzos que ejecutan. “... si el niño durmió o no durmió, si comió o no comió, él SIMCE mide lo más importante: si dos más dos son cuatro y solo eso le interesa...” (Entrevista 7, comunicación personal, 14 de junio 2010), señala otro director, que no comulga con este sistema de medición, optando por focalizarse en los casos más críticos y restar importancia a las que según él, son lecturas mal intencionadas de esta evaluación estandarizada. Al respecto, es preciso agregar que detrás de la lógica de exhibición de los resultados del SIMCE, está la intención de hacer competir a las escuelas, estigmatizando las de peor rendimiento, que son en su mayoría públicas y pertenecientes al perfil socioeconómico más desfavorecido de la población (Pino, Oyarzun y Salinas, 2016).

\subsection{La escuela, una instancia de formación docente}

La profesión docente en Chile lleva más de 50 años apostando por el perfeccionamiento para conseguir la efectividad escolar y enfrentarse a los nuevos desafíos profesionales que exige la educación, instaurándose como una política de carácter permanente (MINEDUC, 2008). Las modalidades varían de acuerdo con las necesidades de la escuela, del sistema y de la iniciativa personal. Las instancias de formación que apuestan por la mejora escolar abarcan más allá de los cursos programados desde dentro y fuera de la institución y la formación docente también puede ser concebida a través de otros mecanismos de análisis y prácticas pedagógicas entre pares.

En el contexto específico de las escuelas de la comuna de Coyhaique, la práctica escolar es también reconocida por los encargados de escuelas como un mecanismo de formación; por tanto, los esfuerzos de la organización se orientan a potenciar el análisis de prácticas colectivas y a propiciar espacios para la interacción entre pares. Como señala una docente: "yo creo que los profesores tenemos que sentir que vamos al colegio no solamente a enseñar, sino también vamos mucho a aprender” (Entrevista 1, comunicación personal, 15 de junio de 2009).

La voluntad del cuerpo docente por formarse, por seguir aprendiendo y por mejorar las prácticas educativas que se ejecutan en la escuela, muchas veces, está condicionada por el medio donde se desenvuelven, por los requerimientos de las necesidades de aprendizaje y por los resultados académicos del alumnado. Por esta razón, el análisis institucional de las obligaciones formativas docentes compete a todo el establecimiento y no solo al profesor. En este punto, el conflicto está en creer que solo es "el profesor el que tiene que cambiar" (Entrevista 6, comunicación personal, 24 de junio de 2009), como señala un directivo. Las demandas de perfeccionamiento si no se analizan con todos los integrantes de la escuela y/o la comunidad educativa, pueden resultar insuficientes y desconectadas de la realidad local.

Por otra parte, las y los docentes son críticos a la hora de calificar las formaciones que se les han brindado: dicen estar cansados de las malas experiencias formativas, de las materias repetidas y de las metodologías poco innovadoras. En el caso de las escuelas rurales, donde la mayoría de las y los docentes no han sido preparados para esa realidad (aulas multigrado y unidocente), se encuentran con un escenario complejo "a mi parecer, es como que se estudia otra carrera dentro de la sala, con realidades que distan mucho de lo que uno aprendió o que en la teoría puede encontrar" (Entrevista 7, comunicación personal, 14 de junio 2010). Aún cuesta que se conciba el aula como un espacio de experimentación y adaptación profesional, para transformarla en una experiencia de aprendizaje para el propio docente. Aquí otro ejemplo de problemas derivados de la formación tradicional y de la escasa formación relacionada con la enseñanza en escuelas rurales:

Tienes tres cursos (cursos combinados) y equé haces tú en la escuela rural? Tienes niños que no saben nada, pedagógicamente hablando, de primero (básico), de segundo (básico) que están ahí y de tercero (básico) que están más avanzados y ¿cómo mezclo los tres? Eso no te lo enseñan en la universidad, te lo enseña la práctica, el ensayo y el error: Hoy día, me salió una 
actividad más o menos bien, bueno eso lo tengo que repetir mañana, pero algo que me salió mal, tengo el deber de reformularlo y avanzar. (Entrevista 6, comunicación personal, 24 de junio de 2009)

En palabras de Boix y Bustos (2014) esto "que pudiera parecer en principio un desequilibrio o complejidad en el planteamiento didáctico y organizativo de estas aulas, incorpora un cúmulo de cuestiones que se relacionan con la diversidad, la sociabilidad y el aprendizaje (p. 30)". La escuela rural, como una instancia de aprendizaje, puede llegar a ser muy provechosa en la planificación de estrategias de enseñanza, como señala un docente: "la parte rural, al tener menos niños, te da un chance de explorar más ámbitos" (Entrevista 3, comunicación personal, 17 de junio de 2009). Estas características son las que permiten generar conocimiento para intercambiar en espacios de conversación y reflexión. En este sentido, existe consciencia de las características especiales y diversas de cada escuela y de que un cambio de mirada respecto al contexto puede tener consecuencias y beneficios tanto profesionales como institucionales.

\subsubsection{Desterritorialización de las politicas educativas}

Algunas escuelas denuncian que algunas medidas de las políticas educativas nacionales pueden transformarse en verdaderos obstáculos para la eficacia de estas. Se mencionan, algunos cambios impuestos por el MINEDUC, sin una clara justificación del por qué; también, se manifiestan la complejidad de trabajar con muchos niveles educativos a la vez, reto para las zonas rurales que acarrea la consecuencia de pensar las aulas multigrado como una desventaja.

En el caso de las escuelas rurales municipalizadas de Coyhaique, el promedio de alumnos/as por curso es entre 4 y 9 estudiantes (MINEDUC, 2008). Desde la centralidad no se promueve la experimentación pedagógica y los directores manifiestan que el tema de los cursos multigrados sigue siendo complejo, al no sentirse con las herramientas adecuadas para enfrentar cursos con diferentes edades y necesidades de aprendizaje y a esto se le suma el hecho de no contar con una red de apoyo docente y el no poder acceder a formación focalizada en esas áreas. La inseguridad en estas materias se contrasta con la práctica y es en esta compleja realidad de aula donde el cuerpo docente termina formándose.

Por otra parte, las y los directivos pierden autonomía en la toma de decisiones respecto a la programación curricular que deben seguir y aplicar, como se aprecia en el siguiente relato:

Esto ocurrió con el plan de 'Apoyo compartido' que era una continuación, pero con distinto nombre, del programa de lectura. El plan de apoyo compartido lo elaboró el gobierno y dijo que se aplicará en la escuela tanto...en la zona tanto...sin preguntarnos al director ni a la unidad educativa, por qué nosotros tenemos que aplicarlo sin saber en qué consistía. (Entrevista 4, comunicación personal, 18 de junio de 2009)

El problema que arguyen algunas personas directivas también tiene que ver con la poca valoración que existe hacia la carrera directiva. No existe una verdadera formación para ser director, no hay ningún sustento formativo, dice un director: "para ser director basta con haber hecho clases en un instituto dos años y vas a ser director...” (Entrevista 7, comunicación personal, 14 junio 2010). Además, manifiesta molestia al comentar que ahora todos creen que saben de educación por el solo hecho de haber cursado una enseñanza obligatoria y eso desprestigia la labor de los que trabajan en el gremio. De acuerdo con esa afirmación, cabe señalar que, para el caso chileno, "las políticas orientadas al liderazgo directivo escolar han desarrollado incipientemente las temáticas de la formación y desarrollo profesional de los directivos” (Donoso y Benavides, 2018, p. 8)

También se manifiesta la preocupación existente por la desaparición inminente de las escuelas municipales, dado el alto número de colegios particulares en detrimento de los municipales, que son quienes atienden a la población más vulnerable de la comuna. En consecuencia, la población que asiste a las escuelas rurales son las más afectadas socioculturalmente cuando existe un cierre inminente. "Los sujetos, tanto adultos como niños, se sienten marginados de un proyecto país que parece no considerar sus demandas" (Núñez, Peña, Cubillos y Solorza, 2016, p. 953). Esta realidad es confirmada por el encargado de una escuela rural: “...hay 
un momento en que nosotros vamos a desaparecer y cuando desaparezcan las escuelas municipales, no sé qué tan importante será considerado el trabajo de hoy (Entrevista 7, comunicación personal, 14 de junio 2010).

\subsection{Escuela abierta al exterior}

La precaria comunicación entre algunas escuelas rurales se justifica aludiendo a la distancia que las separa. Las escuelas rurales están y se sienten más aisladas, también en lo operativo, que se expresa al solicitar algún tipo de ayuda o favorecer algún intercambio de tipo pedagógico. Existe una conciencia sobre los beneficios de la comunicación entre escuelas, que se expresa en la necesidad de mejorar y facilitar los vínculos entre ellas. Al respecto se señala: "las escuelas están distantes y no hay forma de comunicarse. Si uno quisiera una ayuda pronto, no tenemos la forma de comunicarnos" (Entrevista 1, comunicación personal, 15 de junio de 2009).

Las escuelas rurales en Chile no cuentan con espacios organizados, ni institucionalizados, para discutir y analizar el sentido pedagógico de la escuela rural. Es decir, las escuelas rurales trabajan, en su mayoría, como unidades aisladas en un entorno rural, donde puede darse o no una dinámica de relación e intercambio con la comunidad. El MINEDUC cuenta con un Programa de Educación Rural, que dispone de instancias de apoyo al desarrollo de las y los docentes en territorios rurales y en la línea de la actualización y asesoría técnica en aspectos curriculares y pedagógicos. Al ser espacios brindados por la institucionalidad educativa, con el fin de reforzar y reafirmar las políticas educativas nacionales, muchas veces, no prosperan como espacios para el pensamiento y la reflexión de los propios proyectos educativos y como tal, no se conciben como espacios de comunicación e intercambio, sino solo como espacios de formación unidireccional.

Los jefes técnicos de las escuelas urbanas señalan que el apoyo a las escuelas rurales siempre se hace en situaciones muy puntuales. Las escuelas situadas en un contexto de poca densidad demográfica mantienen siempre la demanda latente de establecer alianzas entre escuelas de manera sistemática y permanente. Tal y como señala una directora: "nosotros continuamente estamos consultándonos cosas, pero todavía no hemos establecido una red formal con un establecimiento, ni vamos a hacer pasantías de una escuela a otra ni hacemos actividades conjuntas" (Entrevista 3, comunicación personal, 17 de junio de 2009).

Ante este escenario, sigue primando la interacción de manera informal y puntualizada:

Oye mira: cómo trataste el SIMCE, de qué manera lo viste, de qué manera lo analizaste, de qué manera lo preparaste y cómo hiciste el taller de convivencia que hubo. Este aspecto cómo lo ves tú, cómo lo trabajaste o qué entendiste de esto. (Entrevista 4, comunicación personal, 18 de junio de 2009)

\section{Otro director comenta:}

Y aparte de la formalidad que hay, ante cualquier duda está el teléfono, está la posibilidad de venir a la escuela a preguntarle al jefe técnico, a preguntarle al director, a la orientadora, a la profesora especialista en lenguaje y ellos siempre están dispuestos, siempre con buena voluntad. (Entrevista 7, comunicación personal, 14 de junio 2010)

El tipo de comunicación que permite el sistema de educación municipal es precario y las iniciativas de conexión entre escuelas corren por voluntad o interés de directivos o docentes que necesiten de ese vínculo o resolver algún problema puntual, como lo confirma otro testimonio:

Una vez al mes, el director cita a una reunión, pero estamos vinculados solo en esa reunión. Terminamos la reunión y cada uno va a su negocio. No hay otras reuniones en el año y podemos no vernos nunca más. (Entrevista 8, comunicación personal, 15 de junio 2010)

También se reconoce que el avance en las telecomunicaciones ha permitido una mayor inmediatez en los contactos entre escuelas, considerando el difícil contexto geográfico y climático donde se sitúan las escuelas de Coyhaique. Sumado a esto, el aumento de cursos de perfeccionamiento impulsados por la municipalidad $\mathrm{u}$ otras instituciones ha afianzado los lazos y la comunicación entre escuelas, como comenta una directora: 
Nos proponemos tener mejores relaciones, porque antes éramos islas. Hoy día, creemos que de verdad deberían existir redes entre nosotros y ser más humildes, no estar tan aislados, no ser más egoístas y ser más colaborativos, más solidarios. Hay que profundizar esos valores, manteniéndolos, ya que es la única forma de avanzar. Si se aísla uno, se generan más problemas y no se resuelven bien. Yo, constantemente, con uno o dos en particular converso telefónicamente y diariamente y nos contamos nuestras historias, nuestros problemas: mira yo tengo este problema... (Entrevista 9, comunicación personal, 16 de junio 2010)

Las escuelas tienen la necesidad de comunicarse y mantener un contacto con otras escuelas, posibilitando el traspaso y el intercambio de conocimiento. Como bien decía una directora, son escuelas hermanas que manejan prácticamente los mismos códigos y tienen muchos puntos en común; por ello, es importante que exista una comunicación entre ellas y también hacia la comunidad.

\subsubsection{La escuela como canal de todas las demandas}

La administración de las escuelas es compleja. Seis de los nueve entrevistados concordaron en que la parte administrativa de las escuelas es una tarea engorrosa a la hora de dirigir un establecimiento. Las escuelas urbanas deben coordinar el trabajo curricular de primero básico a cuarto medio, los niveles parvularios, los proyectos de integración permanente y los cursos de lenguaje. En el caso de la escuela rural, un director dice que le falta personal y se queja de que toda la responsabilidad de la escuela recae en la figura del director. Además, las escuelas rurales tienen que supervisar el internado, el tema de las comidas, las salidas, el transporte para el alumnado. El tiempo que se ocupa en las cosas administrativas le quita espacio al resto de las labores propiamente pedagógicas. Un claro ejemplo es el de una directora que, por no contar con internet en su escuela, debe tomarse un día entero laborable para ejecutar trámites administrativos en el departamento de educación municipal de la ciudad, teniendo que suspender el funcionamiento de la escuela por ese día. A lo anterior se suma que la mayoría de los proyectos que elaboran instituciones externas en beneficio de la comunidad pasan por la escuela municipal, es decir, se planifican y se elaboran proyectos de otras organizaciones para ser ejecutado por las escuelas. Esto debería ser visto como un beneficio para las escuelas, siempre y cuando, esos proyectos los ejecuten y desarrollen los propios demandantes. Pero la responsabilidad de aplicarlos recae directamente en la institución educativa y más específicamente en la figura del encargado:

Ni siquiera nos han consultado a nosotros si queremos participar y si lo que ellos están implementando nos sirve o no nos sirve, o si lo estamos trabajando o no lo estamos trabajando: Todo el mundo elabora proyectos para que los ejecuten las escuelas y nos bombardean de aquí y nos bombardean de allá, nos bombardea salud, nos bombardea educación. ¡Oye! no hay institución que no quiera que las escuelas hagan las cosas aquí (Entrevista 2, comunicación personal, 16 de junio 2009).

Por todo lo anterior, una aplicación más flexible de las políticas educativas favorecería la posibilidad de que el propio centro educativo lograra establecer la discusión sobre las necesidades locales. Discriminando entre las iniciativas que podrían o no contribuir a una mejora, se podría focalizar más la intervención. En este sentido, se hace eco de que "un cambio real debe combinar las iniciativas de los centros y el apoyo del sistema educativo, considerando importante la existencia de propuestas planificadas y percibidas como necesarias por los afectados" (Gairín y Rodríguez, 2011, p. 33). Esta podría ser la clave para definir cuáles son los limitantes que no permiten llevar adelante el cambio institucional y de esta manera articular estrategias para encaminarlo.

\section{DisCuSIONES Y CONCLUSIONES}

Esta aportación significó una larga e importante inmersión en el estudio de la realidad de las escuelas públicas de una parte de la patagónica chilena, muchas veces excluida en la toma de decisiones a nivel nacional y ajena 
a las grandes ideas que engloban las reformas educativas. A partir de aquí, se ha generado un conocimiento explícito de esa realidad concreta, que se espera pueda orientar las decisiones educativas generales y específicas.

Se comparte con Donoso que la región de Aysén requiere de una política educacional fuertemente contextualizada a su realidad, que dé cuenta de las particularidades que le impone el territorio y su geografía a la distribución poblacional (Donoso, Arias, Cancino y Oyarzún, 2010, p. 87). Para ello, es necesario conocer y profundizar sobre las dinámicas escolares implicadas en los procesos de mejora educativa como una forma de diagnosticar la complejidad en la que se desarrolla el quehacer educativo patagónico. La literatura sobre el cambio educativo, en este sentido, aporta elementos para el análisis de la institución escolar y para encausar las medidas a considerar en esta materia.

Los supuestos sobre el cambio educativo aportan elementos teóricos y experiencias que pueden influir efectivamente en el desarrollo institucional de las escuelas, a saber, uno de los más recurrentes es situar el foco principal en los procesos y resultados de la enseñanza. Para Pascual (2011), todas las innovaciones poseen un elemento en común: "ponen como elemento final los resultados de aprendizaje de sus alumnos" (p. 30). Así mismo afirman Krichesky y Murillo (2011) cuando señalan que toda la comunicación y el intercambio entre los profesionales deben estar siempre enfocados a los resultados de aprendizaje de estudiantes y asegurarse de que estén efectivamente aprendiendo. Sin embargo, en la práctica subsiste uno de los grandes retos en cuanto a la búsqueda de una manera eficaz de mejorar los resultados de la enseñanza y el aprendizaje en contextos geográficamente periféricos. Si bien hay coincidencia en que el foco de la mejora son los resultados del aprendizaje, la reflexión profunda sobre qué implica este desafío en cada contexto educativo podría marcar la diferencia entre un análisis castigador sobre estos resultados o un análisis reflexivo sobre cómo abordar medidas conjuntas para mejorar los resultados de la enseñanza.

Otro planteamiento teórico del cambio educativo, releva al cuerpo docente como un colectivo de profesionales de una institución educativa, capaz de tomar decisiones propias para la mejora de sus escuelas, en el caso de las escuelas de Coyhaique la principal limitante a la hora de emprender una mejora, es la atención y la importancia que dan las escuelas y su entorno a los resultados escolares, en términos de éxito o fracaso, acción que repercute directamente en el cuerpo docente, es decir, su praxis se ve restringida fuertemente por una política educativa centralista. En este caso concreto las medidas centradas en mejorar los resultados de enseñanza y aprendizaje, serán condicionantes de mejora, siempre y cuando se consideren como un vehículo para posibilitar otras actividades extraescolares, como en el caso de Coyhaique: participar de la orquesta juvenil.

Otra posibilidad de mejora es propiciar espacios de reflexión compartida dentro de la escuela. Esto implica potenciar los espacios de análisis y reflexión conjunta para lograr y hacerse consciente de aquellos mecanismos arraigados en la estructura del sistema educativo chileno que impiden un ejercicio de autonomía, viabilizando mecanismos de gestión desde la propia escuela. Para ello, es preciso considerar la institución educativa como un espacio posible para la organización y la formación, en aquellas materias que son propias del contexto y que solo se llegan a conocer a través de la interacción con la propia realidad escolar.

La mejora escolar está en función de un conjunto de factores, que, por una parte, se ve limitada en escuelas que están bajo un régimen educativo con rasgos centralizadores y homogeneizantes y qué considera las políticas educativas lejanas y, a veces, contrarias a las necesidades locales. Pero que, por otro lado, tiene posibilidades cuando se combinan acciones que focalizan los procesos de enseñanza-aprendizaje con un desarrollo curricular propio y una evaluación pertinente al contexto de actuación.

Lo anterior también ratifica en gran medida que las teorías del cambio educativo entregan elementos de análisis concretos sobre la práctica educativa, pero es responsabilidad de cada institución el considerarlos y adaptarlos de acuerdo a sus necesidades. Lo dicho, es corroborado por personal directivo y docentes de las escuelas de Coyhaique, al plantear que no puede haber un cambio educativo sin actitudes favorables del profesorado hacia este y que, cuando no las hay, lo primero que se ha de conseguir es modificarlas a través de la mejora en los flujos de comunicación, conociendo la experiencia de diversas personas e instituciones que ya 
han transitado por procesos de cambio, además de dialogar y reflexionar sobre las experiencias propias como una manera de abrirse a la comunidad.

Para finalizar, es importante recalcar que los estudios que se desarrollen en educación deberían ser completados y comparados siempre con la realidad. Supone esto aumentar los estudios contextualizados y cualitativos que den a conocer experiencias locales mediante los testimonios de los propios actores, directivos/ as, profesores/as, apoderados/as, alumnos/as y vecinos/as de las escuelas. La finalidad sigue siendo mejorar la educación para mejorar significativamente la vida de las personas, más allá de las cifras que indican éxito o fracasos.

\section{REFERENCIAS}

Atria, F. (2009). ¿Qué educación es "pública”?. Revista de Estudios Sociales, 117, 45-69.

Bernstein, B. (1990). Poder, educación y conciencia. Sociología de la transmisión cultural. España: El Roure Editorial, S.A.

Boix, R, y Bustos, A. (2014). La enseñanza en las aulas multigrado. Una aproximación a las actividades escolares y los recursos didácticos desde la perspectiva del profesorado. Revista Iberoamericana de Evaluación Educativa, 7(3), $29-43$.

Bolívar, A. (2004). Los centros educativos como organizaciones que aprenden. Promesas y realidades. Madrid: Aula Abierta.

Bourdieu, P., y Passeron, J. C. (1996). La Reproducción. Elementos para una teoría del sistema de enseñanza. Barcelona, Madrid: Editorial Laia.

Bürgi, J. y Peralta, M. (2011). El Concepto de Calidad Educativa en las Investigaciones sobre Educación en Chile (2000-2008). REICE. Revista Iberoamericana sobre Calidad, Eficacia y Cambio en Educación, 9(3), 72-93. Recuperado de http://www.rinace.net/reice/numeros/arts/vol9num3/art4.pdf

Donoso, S. y Benavides, N. (2018). Prácticas de gestión de los equipos directivos de escuelas públicas chilenas. Revista Brasileira de Educacao. 23, 1-28. doi: 10.1590/s1413-24782018230013

Donoso, S., Arias, O., Cancino, V. y Oyarzún, C. (2010). Desarrollo educacional y territorio en la región de Aysén. En J. Gairín y D. Castro (Eds.), Desarrollo Educativo al servicio del desarrollo social. Proyecto de apoyo y fortalecimiento educacional en gestión directiva y competencias profesionales docentes (pp. 71-90). Santiago, Chile: Troncoso Impresores.

Escudero, J. M. (2001). La mejora de la educación como marco de referencia para el asesoramiento pedagógico. En J. D. Segovia (Coord.), Asesoramiento al centro educativo. Colaboración y cambio en la institución (pp. 15-50). Barcelona, España: Octaedro.

Fullan, M (2002a). El significado del cambio educativo. Un cuarto de siglo de aprendizaje. Profesorado, revista de curriculum y formación del profesorado, 6(1-2), 1-14.

Fullan, M (2002b). Los Nuevos Significados Del Cambio En La Educación. Barcelona: Ediciones Octaedro.

Gairín, J. (2015). Las comunidades de práctica profesional. Una breve Introducción. En J. Gairín (Coord.), Las Comunidades de Práctica profesional. Creación, desarrollo y evaluación. (pp. 13-31). Barcelona: Wolters Kluwer.

Gairín, J. y Castro, D. (Eds). (2010). Desarrollo Educativo al servicio del desarrollo social. Santiago de Chile: FIDECAP.

Gairín, J. y Muñoz, J.L. (2008). El agente de cambio en el desarrollo de las organizaciones. Enseñanza, 26, 187-206.

Gairín, J. y Rodríguez, D. (2011). Cambio y mejora en las organizaciones educativas. Educar 47(1), 31-50.

Gairín, J. y Sánchez, S. (2011). Municipio y Educación. Santiago, Chile: FIDECAP

Guarro, A. (2005). Los procesos de cambio educativo en una sociedad compleja. España: Ediciones Pirámide.

Hargreaves, A. (2003). Replantear el cambio educativo. Un enfoque innovador. Buenos Aires: Amorrortu.

Krichesky G.J. y Murillo, F.J. (2011). Las Comunidades Profesionales de Aprendizaje. Una Estrategia de Mejora para una Nueva Concepción de Escuela. REICE. Revista Iberoamericana sobre Calidad, Eficacia y Cambio en Educación, 9(1), 65-83. 
Mertler, C. (2016). Leading and facilitating educational change through action research learning communities. Jeel, 3(3), 1-12.

Ministerio de Desarrollo Social de Chile. (2011). Resultados Regionales Encuesta CASEN. $N^{\circ} 11$. Recuperado de htt ps://bit.ly/2QQZrdA

Ministerio de Educación de Chile [MINEDUC]. (2008). Indicadores para la Educación. Ministerio de Educación. República de Chile. Santiago, Chile. Recuperado de https://bit.ly/36VzJtR

Miranda, E. (2002). La supervisión escolar y el cambio educativo. Un modelo de supervisión para la transformación, desarrollo y mejora de los centros. Profesorado, revista de curriculum y formación del profesorado, 6(1-6), 1-15.

Mora. G., Monge, P. M., y Santiago, J.M. (2018). Liderazgo para la gestión del cambio en escuela de bajo desempeño. Revista de Investigación Educativa de la Escuela de Graduados en Educación, 8(16), 53-61.

Murillo, F.J. (2011). Mejora de la eficacia escolar en Iberoamérica. Revista Ibero-Americana de educaçâo, 55, 49-83.

Núñez, C.G., Peña, M., Cubillos, F., y Solorza, H. (2016). Estamos todos juntos: el cierre de la Escuela rural desde la perspectiva de los niños. Educ. Pesque., Sao Paulo, 42(4), 953-967.

OCDE (2004). Revisión de politicas nacionales en educación: Chile, París y Santiago: OCDE y MINEDUC. Santiago de Chile.

PADEM (2011). Propuesta General Plan de Desarrollo Comunal 2006-2010. Ilustre Municipalidad de Coybaique. Comuna de Coyhaique. Recuperado de www.coyhaique.cl

Pascual, J. (2011). El Efecto Escuela. Más allá del Aula. REICE. Revista Iberoamericana sobre Calidad, Eficaciay Cambio en Educación, 9(1), 28-45.

Pino, M., Oyarzun, G. y Salinas. I. (2016). Crítica a la rendición de cuentas: narrativa de resistencia al sistema de evaluación en Chile. Cad. Sedes, Campinas, 100, 337-354.

Raczynski, D y Salinas, D. (2008). Aportes y desafíos en la evaluación de políticas y programas de mejoramiento educativo. Reflexiones desde nuestra práctica de evaluación en chile. Revista Iberoamericana de Evaluación Educativa, 1(1), 79-92.

Retamal, S. (2012). Análisis de las condiciones de éxito de un programa de desarrollo educativo. Proyecto: Apoyo y Fortalecimiento Educacional en Gestión Directiva y Competencias Profesionales Docentes. AECID CoyhaiqueChile. (Tesis de Doctorado). Universidad Autónoma de Barcelona, Barcelona-España.

Sánchez, S. (2009). La formación permanente del profesorado centrada en la escuela. Análisis de su Funcionamiento. (Tesis de Doctorado). Universidad Autónoma de Barcelona. Barcelona, España.

Santos, M. A. (2002). La escuela que aprende. Madrid: Morata.

Santos, M. A. (2008). La pedagogía frente a Frankenstein y otros textos frente al desaliento educativo (01 ed. ed.). Barcelona, Madrid: Graó.

Serra, C. (2004). Etnografía escolar, etnografía de la educación. Revista de Educación, 334, 165-176.

Tiana, A. (s.f.). Una mirada técnico-pedagógica acerca de las evaluaciones de la calidad de la educación. Apuntes para el Módulo, calidad, evaluación en Innovación en Educación. España: Universidad Autónoma de Barcelona.

Vera, D., Salvo, S., y Zunino, H. (2013). En torno al cierre de escuelas rurales en Chile. Antecedentes para la implementación de una política de Estado. Revista Investigaciones en Educación, 13(1), 123-143.

\section{Notas}

[1] Este artículo deriva de la tesis doctoral de la autora (Retamal, 2012), titulada Análisis de las condiciones de éxito de un programa de desarrollo educativo. Proyecto: Apoyo y Fortalecimiento Educacional en Gestión Directiva y Competencias Profesionales Docentes. AECID Coyhaique-Chile.

[2] En el marco de un proyecto de apoyo y fortalecimiento educacional en gestión directiva y competencias profesionales docentes, dirigido por Joaquín Gairín (Grupo EDO: http://edo.uab.es) y subvencionado por la Agencia Española de Cooperación Internacional para el Desarrollo (AECID) en la convocatoria de 2007. Ver resultados en Gairín y Castro (2010) y Gairín y Sánchez (2011). 
[3] En el marco de un proyecto de apoyo y fortalecimiento educacional en gestión directiva y competencias profesionales docentes, dirigido por Joaquín Gairín (Grupo EDO: http://edo.uab.es) y subvencionado por la Agencia Española de Cooperación Internacional para el Desarrollo (AECID) en la convocatoria de 2007. Ver resultados en Gairín y Castro (2010) y Gairín y Sánchez (2011)

[4] En el marco de un proyecto de apoyo y fortalecimiento educacional en gestión directiva y competencias profesionales docentes, dirigido por Joaquín Gairín (Grupo EDO: http://edo.uab.es) y subvencionado por la Agencia Española de Cooperación Internacional para el Desarrollo (AECID) en la convocatoria de 2007. Ver resultados en Gairín y Castro (2010) y Gairín y Sánchez (2011).

[5] Cuando se recogieron los datos, aún no se concretaba la instalación de la Universidad de Aysén, institución de educación superior de dependencia estatal.

\section{BY-NC-ND}

